\section{IN BRIEF}

\section{PRIONS}

Species-barrier-independent prion replication in apparently resistant species.

Hill, A. F. et al.

Proc. Natl Acad. Sci. USA 97, 10248-10253 (2000)

Until now, it was believed that a species barrier limits infection by prions from different species. For example, mice cannot be infected with hamster prions but humans can be infected with cow prions. Hill et al. now show that hamster prions can replicate in mice - they just do not cause prion disease. But if either mice or hamsters are inoculated with brain extracts from these sub-clinically infected mice, they do develop the disease.

\section{CELL ADHESION}

Two cell adhesion molecules, nectin and cadherin, interact through their cytoplasmic domain-associated proteins.

Tachibana, K. et al.

J. Cell Biol. 150, 1161-1175 (2000)

Nectin is a calcium-independent immunoglobulin-like adhesion molecule that can trans-interact with itself to form a cell-cell adhesion system, similar to the well-known cadherin system. The nectin and cadherin adhesion complexes interact with each other through their cytoplasmic domain-associated proteins l-afadin and catenins, respectively. Although the two systems cooperate in organizing adherens junctions, their hierarchy is not known.

\section{ALZHEIMER'S DISEASE}

Nicastrin modulates presenilin-mediated notch/g/p-1 signal transduction and $\beta$ APP processing.

$Y u$, G. et al.

Nature 407, 48-54 (2000)

Processing of the $\beta$-amyloid precursor protein ( $\beta \mathrm{APP}$ ) to the amyloid- $\beta$ peptide is central in the path to Alzheimer's disease, and this paper reports the identification of a new player in the process. Called nicastrin, this protein interacts with $\beta A P P$, and also with presenilins (PS) 1 and 2. The authors believe it to be a functional component of the PS1 and PS2 complexes, and show that, like them, nicastrin is involved in processing both $\beta$ APP and Notch.

\section{CELL DIVISION}

Analysis of mid1p, a protein required for placement of the cell division site, reveals a link between the nucleus and the cell surface in fission yeast.

Paoletti, A. \& Chang, F

Mol. Biol. Cell 11, 2757-2771 (2000)

In fission yeast, a contractile ring forms in the middle of the cell in early mitosis, and its position depends on the position of the nucleus. The position of the plane of cell division with respect to the nucleus is probably defined by midlp. This protein shuttles between the nucleus and a broad band at the medial cell surface, but how exactly does it convey the positional information?

\title{
INOSITOL PHOSPHATES
}

\section{From duckweed to DNA...}

What does a tiny aquatic flowering plant have in common with a process for repairing broken DNA molecules? The answer is that both require a phosphorylated cyclic alcohol called inositol hexakisphosphate $\left(\mathrm{Ins}_{6}\right)$, and, for the DNA-repair process at least, a report by Hanakahi and colleagues in the 15 September issue of Cell explains why.

The story revolves around the catalytic subunit of the DNA-dependent protein kinase (DNA- $\mathrm{PK}_{\mathrm{cs}}$ ), which helps to repair double-stranded DNA breaks using the non-homologous end-joining (NHEJ) pathway. The DNA-PK $\mathrm{cs}_{\mathrm{cs}}$ is first targeted to each of the severed ends by a complex of the $\mathrm{Ku} 70$ and Ku 80 proteins (see figure). DNA-PK $\mathrm{C}_{\mathrm{cs}}$ then recruits a complex of DNA ligase IV and XRCC4, resulting in rejoining of the broken ends.

In vitro assays have shown that although all of these players - Ku70, $\mathrm{Ku} 80, \mathrm{DNA}^{-\mathrm{PK}_{\mathrm{cs}}}$, XRCC4 and DNA ligase IV - are needed for NHEJ, they are not sufficient. So something is missing, and to discover what, Hanakahi et al. used an in vitro complementation assay. They took cell-free extracts that could promote NHEJ, fractionated them on a phosphocellulose column, then added back various combinations of the fractions to restore the NHEJ activity. In so doing, the authors identified a fraction containing the elusive factor, which they named stimulatory factor A (SFA).

The task of identifying SFA involved eight purification steps followed by a barrage of tests, including protease digestion, nuclease digestion and even boiling. But the activity survived all of these insults intact, indicating - unexpectedly - that SFA is neither a protein nor a nucleic acid.

So what is it? The authors concluded that the active ingredient is, in

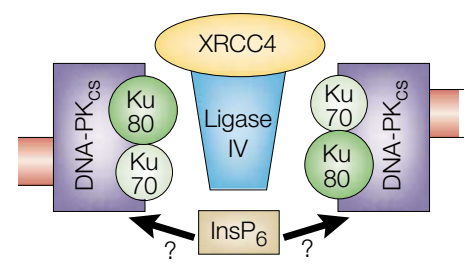

fact, $\operatorname{Ins}_{6}$, and showed that the component of the NHEJ machinery to which it binds is probably DNA- $\mathrm{PK}_{\mathrm{cs}}$ They suggest several possible functions for Ins $\mathrm{P}_{6}$ in NHEJ, including the idea that it converts DNA-PK from an inactive to an active form.

Interestingly, $\mathrm{DNA}-\mathrm{PK}_{\mathrm{cs}}$, as well as several other DNA-repair proteins such as ataxia telangiectasia mutated (ATM) and ATR, contains motifs characteristic of phosphatidylinositol3 -OH kinases $(\mathrm{PI}(3) \mathrm{K})$. As none of these proteins has lipid kinase activity, why have they retained such motifs? Hanakahi et al. speculate that such proteins may have evolved from a common ancestor with both protein and lipid kinase functions, and that mutation of the $\mathrm{PI}(3) \mathrm{K}$ domain caused these proteins to lose their lipid kinase activity but retain the ability to bind headgroups containing inositol polyphosphates. However, there is no evidence that this is where the Ins $\mathrm{P}_{6}$ binds DNA-PK $\mathrm{Cs}_{\mathrm{cs}}$ - indeed, there is a big charge difference between the phosphatidylinositol-3,4,5-trisphosphate headgroup and $\mathrm{InsP}_{6}$ - and it may turn out that $\operatorname{InsP}_{6}$ binds to an allosteric activation site on DNA-PK

Either way, Ins $\mathrm{P}_{6}$ is a molecule of the moment. As well as making up an enormous $60 \%$ of the volume of the aquatic plant duckweed, where it acts as a kind of ballast, $\mathrm{InsP}_{6}$ is thought to act as an antioxidant and phosphate storage source in plant seeds. In mammalian cells it has been implicated in regulating growth, inflammation and nervous transmission, and it is even thought to be involved in export of messenger RNA from the nucleus. Nonetheless, the link to DNA repair comes as a surprise, and should trigger a search for $\mathrm{InsP}_{6}$-binding sites in other members of the PI(3)K family.

\section{Alison Mitchell}

\section{0) References and links}

\section{ORIGINAL RESEARCH PAPER Hanakahi, L. A,}

Bartlet-Jones, M., Chappell, C., Pappin, D. \&

West, S. C. Binding of inositol phosphate to DNA PK and stimulation of double-strand break repair. Cell 102, 721-729 (2000)

REVIEW Smith, G. C. M. \& Jackson, S. P. The DNA-dependent protein kinase. Genes Dev. 13, 916-934 (1999) 